\title{
55 FOODS THAT FUEL PERFORMANCE
}

Carole A Conn Associate Professor of Nutrition, University of New Mexico, Albuquerque, New Mexico, USA

10.1136/bjsm.2010.078725.55

This presentation will discuss the physiological mechanisms that convert food to energy required for powerful performance. Nutrition science will be translated to practical food choices for maximising energy utilisation for training and competition in endurance and resistance sports. Issues to be discussed include the fundamental importance of carbohydrates and hydration, the role of fats in daily training, how proteins optimise tissue growth and repair, and current theories of timing of nutrient intake for optimal competition. Dr Conn is a Board Certified Specialist in Sports Dietetics. She teaches nutrition science and sports nutrition to both undergraduate and graduate students. She provides lectures internationally on the effects of exercise and nutrition for maintaining health and optimising performance. Her published research includes the effects of exercise and nutrition on growth, immune function and chronic disease. She serves as a Fellow of the American College of Sports Medicine. 